\title{
ESTIMATION OF HETEROSIS IN OKRA [ABELMOSCHUS ESCULENTUS (L.) MOENCH] FOR FRUIT YIELD AND ITS COMPONENTS THROUGH LINE $\times$ TESTER MATING DESIGN
}

\author{
ShIVAPPA M KaRAdi* AND CN HaNChinAMANI ${ }^{1}$ \\ Department of Horticulture, Kittur Rani Channamma College of Horticulture, \\ Arabhavi- 591 218, University of Horticultural Sciences, Bagalkote, Karnataka, India
}

Keywords: Heterosis, Mid parent, Better parent, Commercial check, Line $\times$ tester analysis, Okra

\begin{abstract}
Estimation the magnitude of heterosis for yield and yield contributing parameters of Okra was conducted. Fifty-four $F_{1}$ hybrids were generated by line $x$ tester mating design. These $F_{1}$ s along with 21 parents and commercial check (Mhyco-10) were evaluated in a randomized block design with two replications. Analysis of variance with respect to yield and quality characters in parents and hybrids were highly significant. This indicates the existence of high variation in parents and hybrids for different characters studied. Out of 54 cross combinations, seven crosses revealed the significant and positive heterosis over better parent. While 11 crosses showed positive and significant heterosis over economic parent. It is pertinent to mention that the crop has potential to produce the heterotic cross combinations and such crosses can be used for further improvement of this crop. Five crosses, namely L43 $\times$ T44, L22 × T36, L22 × T44, L53 $\times$ $\mathrm{T} 36$ and L31 $\times$ T23 were found to be potential for the production of fruit yield per plant and other desired characters. The high heterosis and per se performance was found in hybrid L43 $\times$ T44 over both better parents and standard check for fruit yield per plant. This indicates that the cross can be exploited commercially.
\end{abstract}

\section{Introduction}

Okra [Abelmoschus esculentus (L.) Moench] belonging to Malvaceae is an important vegetable crop of the tropics and subtropics. It is specially valued for its tender, delicious green fruits which are cooked, canned and consumed in various forms in different parts of the country. India is the largest producer of okra covering an area of 0.50 million hectares with an annual production of 5.85 million tonnes (Anon. 2016a). It is a potential export earner accounting for $13 \%$ of export of fresh vegetables. The ease in emasculation, very high per cent of fruit set and large number of seeds per fruit makes commercial exploitation of hybrid vigour easy in okra. Being an often cross-pollinated crop, out crossing to an extent of 5-9\% by insects is reported which renders considerable genetic diversity Duggi et al. (2013). India is the leading country in okra production, but the yield potential is very low attributing to poor yielding varieties and incidence of various pests and diseases. The low cost of production and high nutritional value have enhanced the usefulness of this crop (Aykroyd 1963). Hybrid breeding has helped in overcoming the yield barriers by accelerating increase in productivity. Hybrid vigour in okra has been first reported by Vijayaraghavan and Warier (1946). Despite high cost of hybrid seeds, there has been an increasing interest among farmers on cultivation of hybrids of this crop. This is mainly because under optimum crop production and protection management, the crops raised from $F_{1}$ hybrids show higher yield due to large size and increased number of fruits per plant.

To initiate an effective breeding programme, it is imperative to have information on various genetic parameters. Since yield potential of okra is generally low the productivity of okra

*Author for correspondence: <shivukaradiphd@gmail.com>. ${ }^{1}$ University of Horticultural Sciences, Bagalkot, Karnataka, India-587 104. 
presumably be increased by improving the genetic architecture through hybridization and recombination. Indeed, knowledge of heterosis of yield and its component characters should be placed on greater emphasis. Hence, the first step in okra improvement should involve evaluation of the germplasm for genetic variability. As a second step, it is required to generate crosses employing a suitable mating design to know the extent of heterosis for various economic traits and inheritance pattern of desired characters, which in turn, would help in deciding the breeding strategies as well as identifying potential parents and crosses for further use in breeding programme (Singh and Singh 2012). The present study was, therefore, aimed to estimate the magnitude of heterosis for yield and yield components of okra through line $\times$ tester mating design.

\section{Material and Methods}

The investigation on heterosis studies in okra was carried out at the Department of Vegetable Science, K.R.C. College of Horticulture, Arabhavi, Gokak Taluk, Belgavi district of Karnataka state during 2016-2017. The experimental material comprised of 18 lines (L-4, L-5, L-6, L-14, L16, L-17, L-22, L-24, L-31, L-37, L-38, L-39, L-42, L-43, L-47, L-50, L-53 and L-65) and three testers (T23, T36 and T44) which were collected from different sources and their $54 \mathrm{~F}_{1}$ hybrids along with one commercial check (MHY-10). Each of the 18 lines were crossed with each of the three testers to derive $54 \mathrm{~F}_{1}$ hybrids. The experiment was laid out in randomized block design with two replications and sowing was done on 24-08-2018. Each treatment or a genotype in each replication was represented by one row each accommodating 15 plants at a row to row spacing of $60 \mathrm{~cm}$ and $30 \mathrm{~cm}$ from plant to plant. Recommended package of practices were i.e., about 25 tonnes of FYM per hectare and the recommended basal dose of fertilizers $(62.5: 75: 62.5 \mathrm{~kg}$ $\mathrm{NPK} / \mathrm{ha}$ ) were incorporated into the soil just before sowing. The remaining $62.5 \mathrm{~kg}$ of nitrogen was applied as a top dress at 45 days after sowing. To raise a good crop and harvesting of fruit was started on 11-10-2018 and up to one and half month of harvesting was done at alternate day .Observations were made on five randomly selected plants in parents, $F_{1} s$ and check in each replication for characters, viz., fruit length, fruit diameter, average fruit weight, number of fruits per plant, fruit yield per plant, fruit yield per plot, fruit yield per hectare, number of ridges on fruit surface, number of seeds per fruit and vitamin $\mathrm{C}$ content. Five plants were randomly selected for each genotype from each replication and evaluated for the quantitative characters and the replicated mean values of various characters of parents and hybrids were subjected to line $\times$ tester $(1 \times t)$ with the method suggested by Kempthorne (1957).

\section{Results and Discussion}

Analysis of variance with respect to ten characters in parents and hybrids revealed that mean sum of squares due to characters in hybrids were highly significant for different characters (Table 1). The variance due to genotypes, crosses and line $\times$ tester showed significant differences for all the characters. Heterosis for yield and quality parameters are presented in Table 2 . For fruit length the cross L22 $\times$ T36 showed maximum and positive significant heterosis over mid parent (32.09\%), better parent (29.19\%) and over the commercial check (53.41\%). Out of 54 crosses, 15 crosses over mid parent, 11 crosses over better parent and 46 crosses over commercial check recorded positive and significant heterosis (Table 2). The findings were at par with the expectation because the significant relative heterosis for these characters have direct influence on yield as also reported earlier by Thippeswamy (2001), Shoba and Mariappan (2005), Kishor et al. (2013), Ashwani et al. (2013) and Neetu et al. (2015) in okra.

For fruit diameter the maximum positive and significant heterosis over mid parent $(40.85 \%)$ and better parent $(35.12 \%)$ was exhibited by the cross L47 $\times$ T36 and the cross L22 $\times$ T44 (22.12\%) over commercial check. Among 54 crosses, 36 crosses over mid parent, 23 crosses over 


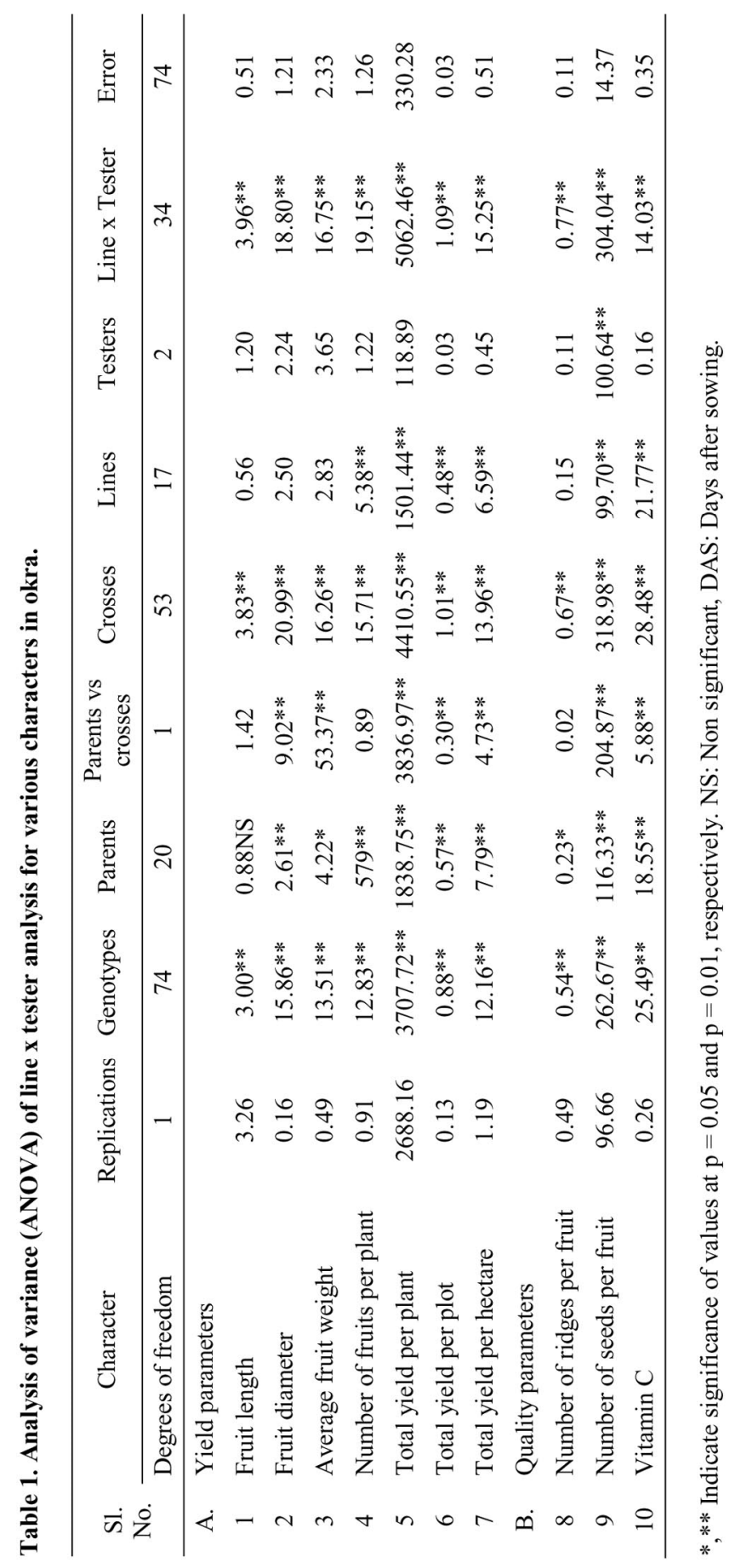




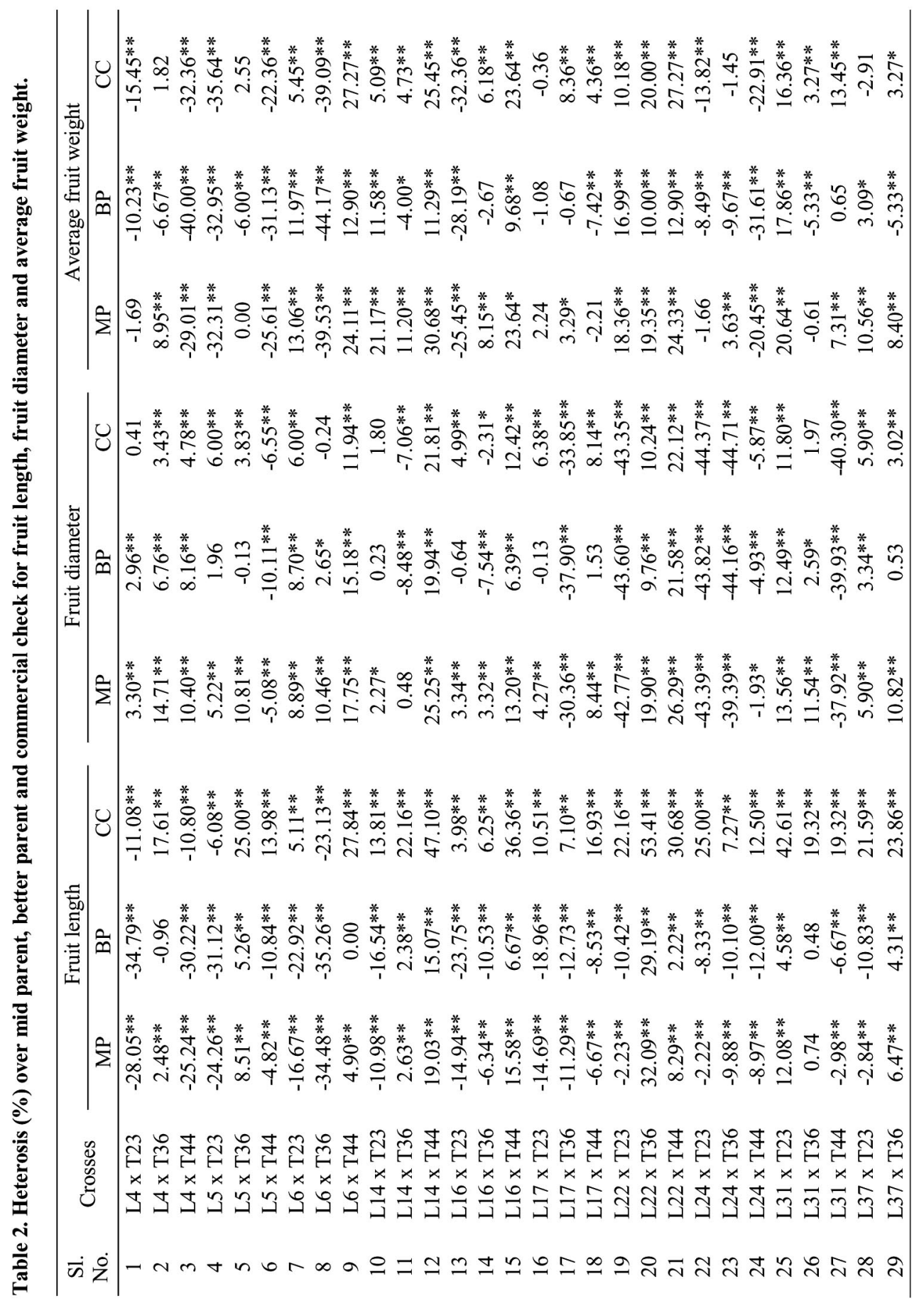




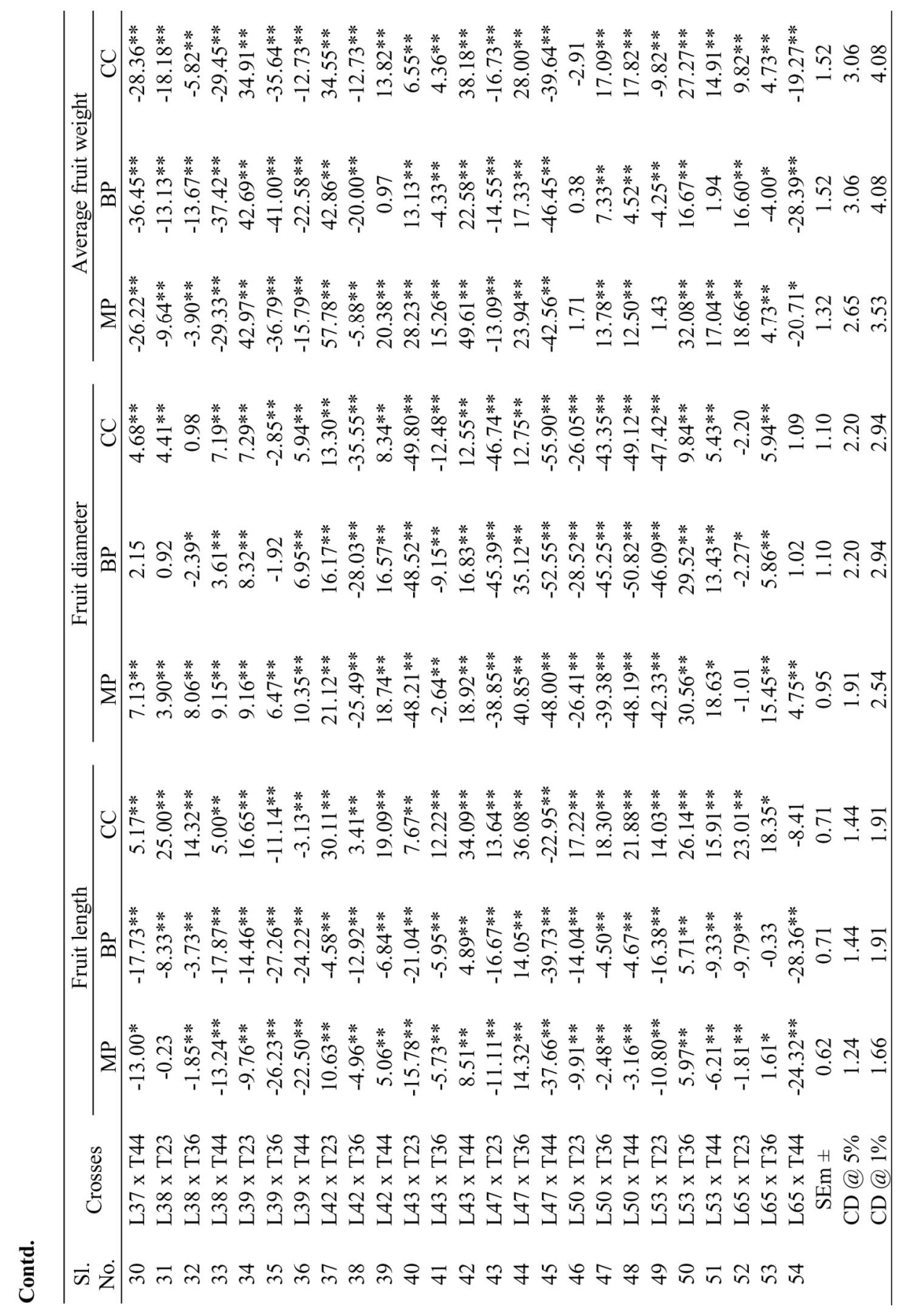


better parent and 28 crosses over commercial check exhibited significant and positive heterosis. For average fruit weight among 54 crosses, 30 crosses over mid parent, 19 crosses over better parent and 28 crosses over commercial check. These findings are in agreement with the findings of Jindal et al. 2009, Ashwani et al. (2013), Verma and Sood (2015) and Sabesan et al. (2016) in okra. Magnitude of heterosis was significant in both the directions over mid parent, better parent and commercial check.

For number of fruits per plant the maximum and positive significant heterosis was observed in the cross L43 x T44 over mid parent $(71.72 \%)$ and over better parent $(47.83 \%)$ and the cross L22 x T36 over commercial check (75.00\%) (Table 3). Among 54 crosses, 13 crosses over mid parent, 11 crosses over better parent and 24 crosses over commercial check exhibited significant and positive heterosis. Plausibly the involvement of non-additive (dominance) gene action was very important for expression of these traits, as also revealed in the reports of Thippeswamy (2001) and Khanapara et al. 2009 in okra.

Table 3. Heterosis (\%) over mid parent, better parent and commercial check for number of fruits/plant and yield /plant.

\begin{tabular}{|c|c|c|c|c|c|c|c|}
\hline \multirow{2}{*}{$\begin{array}{l}\text { Sl. } \\
\text { No. }\end{array}$} & \multirow{2}{*}{ Crosses } & \multicolumn{3}{|c|}{ Number of fruits per plant } & \multicolumn{3}{|c|}{ Fruit yield per plant } \\
\hline & & MP & $\mathrm{BP}$ & $\mathrm{CC}$ & MP & $\mathrm{BP}$ & $\mathrm{CC}$ \\
\hline 1 & $\mathrm{~L} 4 \times \mathrm{T} 23$ & $-8.37 * *$ & $-20.90 * *$ & 1.25 & 2.65 & $-9.24 *$ & $-10.34 *$ \\
\hline 2 & $\mathrm{~L} 4 \times \mathrm{T} 36$ & $-3.60 * *$ & $-17.05 * *$ & $7.00 * *$ & -4.29 & $-16.24 * *$ & $-15.28 * *$ \\
\hline 3 & $\mathrm{~L} 4 \times \mathrm{T} 44$ & $-16.35 * *$ & $-24.35 * *$ & $-13.00 * *$ & $-27.03 * *$ & $-37.26 * *$ & $-33.83 * *$ \\
\hline 4 & L5 $x$ T23 & $-29.55^{* *}$ & $-39.45 * *$ & $-22.50 * *$ & $-20.18 * *$ & $-26.36 * *$ & $-27.24 * *$ \\
\hline 5 & L5 x T36 & $-6.33 * *$ & $-19.77 * *$ & $3.50 * *$ & 6.22 & -3.04 & -1.93 \\
\hline 6 & L5 x T44 & $16.91 * *$ & $5.22 * *$ & $21.00 * *$ & 8.04 & -3.21 & 2.09 \\
\hline 7 & L6 x T23 & $-12.02 * *$ & $-12.69 * *$ & $13.50 * *$ & -4.59 & -5.63 & -4.70 \\
\hline 8 & L6 x T36 & $-41.31 * *$ & $-41.54 * *$ & $-24.00 * *$ & $-54.06 * *$ & $-54.09 * *$ & $-53.57 * *$ \\
\hline 9 & L6 x T44 & $26.53 * *$ & $19.23 * *$ & $55.00 * *$ & $10.59 * *$ & 8.24 & $14.16^{* *}$ \\
\hline 10 & $\mathrm{~L} 14 \times \mathrm{T} 23$ & $-19.64 * *$ & $-30.47 * *$ & $-11.00 * *$ & -4.25 & $-15.56 * *$ & $-16.58 * *$ \\
\hline 11 & L14 x T36 & $-51.91 * *$ & $-58.53 * *$ & $-46.50 * *$ & $-38.19 * *$ & $-46.04 * *$ & $-45.42 * *$ \\
\hline 12 & L14 x T44 & $34.29 * *$ & $21.74 * *$ & $40.00 * *$ & $23.77 * *$ & 6.16 & $11.96 * *$ \\
\hline 13 & L16 x T23 & $-9.47 * *$ & $-14.06 * *$ & $10.00 * *$ & 1.72 & -7.58 & -8.69 \\
\hline 14 & L16 x T36 & $-26.64 * *$ & $-30.62 * *$ & $-10.50 * *$ & -1.01 & $-11.00 *$ & $-9.99 *$ \\
\hline 15 & L16 x T44 & $17.39 * *$ & $17.39 * *$ & $35.00 * *$ & $27.34 * *$ & $12.41 * *$ & $18.55 * *$ \\
\hline 16 & $\mathrm{~L} 17 \times \mathrm{T} 23$ & $-3.46 * *$ & $-18.36 * *$ & $4.50 * *$ & $11.99 * *$ & 3.56 & 2.31 \\
\hline 17 & L17 x T36 & -0.69 & $-16.28 * *$ & $8.00 * *$ & $18.59 * *$ & 8.49 & $9.73^{*}$ \\
\hline 18 & L17 x T44 & $6.63 * *$ & $-5.65 * *$ & $8.50 * *$ & $8.97 * *$ & -2.16 & 3.18 \\
\hline 19 & $\mathrm{~L} 22 \times \mathrm{T} 23$ & $-33.19 * *$ & $-40.63 * *$ & $-24.00 * *$ & $-24.69 * *$ & $-27.91 * *$ & $-28.78 * *$ \\
\hline 20 & L22 x T36 & $53.17 * *$ & $35.66 * *$ & $75.00 * *$ & $33.00 * *$ & $25.90 * *$ & $27.33 * *$ \\
\hline 21 & $\mathrm{~L} 22 \times \mathrm{T} 44$ & $49.18 * *$ & $39.13 * *$ & $60.00 * *$ & $25.57 * *$ & $16.57 * *$ & $22.94 * *$ \\
\hline 22 & $\mathrm{~L} 24 \times \mathrm{T} 23$ & $-34.71 * *$ & $-41.21 * *$ & $-24.75 * *$ & $-19.26 * *$ & $-25.00 * *$ & $-25.91 * *$ \\
\hline 23 & L24 x T36 & $-14.04 * *$ & $-22.87 * *$ & -0.50 & $-15.44 * *$ & $-22.29 * *$ & $-21.41 * *$ \\
\hline 24 & $\mathrm{~L} 24 \times \mathrm{T} 44$ & $-10.80 * *$ & $-15.65 * *$ & $-3.00 *$ & $-11.87 * *$ & $-20.52 * *$ & $-16.18 * *$ \\
\hline 25 & $\mathrm{~L} 31 \times \mathrm{T} 23$ & $24.51 * *$ & $24.03 * *$ & $60.00 * *$ & $25.30 * *$ & $23.33 * *$ & $21.84 * *$ \\
\hline 26 & L31 x T36 & $-17.44 * *$ & $-17.44 * *$ & $6.50 * *$ & 1.65 & -1.09 & 0.04 \\
\hline 27 & $\mathrm{~L} 31 \times \mathrm{T} 44$ & $-28.28 * *$ & $-32.17 * *$ & $-12.50 * *$ & $-22.45 * *$ & $-26.04 * *$ & $-22.00 * *$ \\
\hline
\end{tabular}


Contd.

\begin{tabular}{|c|c|c|c|c|c|c|c|}
\hline \multirow{2}{*}{$\begin{array}{l}\text { Sl. } \\
\text { No. }\end{array}$} & \multirow[t]{2}{*}{ Crosses } & \multicolumn{3}{|c|}{ Number of fruits per plant } & \multicolumn{3}{|c|}{ Fruit yield per plant } \\
\hline & & MP & BP & $\mathrm{CC}$ & MP & BP & $\mathrm{CC}$ \\
\hline 28 & L37 x T23 & $-22.92 * *$ & $-23.83 * *$ & $-2.50 *$ & $15.87 * *$ & $-12.53 * *$ & $-13.59 * *$ \\
\hline 29 & L37 x T36 & $-25.59 * *$ & $-26.74 * *$ & $-5.50 * *$ & 2.28 & $-23.40 * *$ & $-22.52 * *$ \\
\hline 30 & L37 x T44 & $-23.75 * *$ & $-26.80 * *$ & $-8.50 * *$ & -0.90 & $-26.79 * *$ & $-22.79 * *$ \\
\hline 31 & $\mathrm{~L} 38 \times \mathrm{T} 23$ & $14.01 * *$ & $-7.81 * *$ & $18.00 * *$ & 7.93 & 4.44 & 3.18 \\
\hline 32 & L38 x T36 & $-25.96 * *$ & $-40.31 * *$ & $-23.00 * *$ & $-34.73 * *$ & $-37.55 * *$ & $-36.84 * *$ \\
\hline 33 & $\mathrm{~L} 38 \times \mathrm{T} 44$ & $-31.96 * *$ & $-42.61 * *$ & $-34.00 * *$ & $-26.13 * *$ & $-30.70 * *$ & $-26.92 * *$ \\
\hline 34 & L39 x T23 & $-18.42 * *$ & $-27.34 * *$ & $-7.00 * *$ & $11.02 * *$ & -0.02 & -1.23 \\
\hline 35 & L39 x T36 & $-30.57 * *$ & $-38.37 * *$ & $-20.50 * *$ & $-25.52 * *$ & $-33.62 * *$ & $-32.86 * *$ \\
\hline 36 & L39 $\times$ T44 & -1.86 & $-8.26^{* *}$ & $5.50 * *$ & $9.41 * *$ & -4.25 & 0.99 \\
\hline 37 & $\mathrm{~L} 42 \times \mathrm{T} 23$ & $43.48 * *$ & $28.91 * *$ & $65.00 * *$ & $41.96 * *$ & $20.00 *$ & $18.55 * *$ \\
\hline 38 & $\mathrm{~L} 42 \times \mathrm{T} 36$ & $-30.74 * *$ & $-37.98 * *$ & $-20.00 * *$ & $-41.80 * *$ & $-51.27 * *$ & $-50.71 * *$ \\
\hline 39 & $\mathrm{~L} 42 \times \mathrm{T} 44$ & $-13.82 * *$ & $-18.70 * *$ & $-6.50 * *$ & $-10.67 * *$ & $-26.44 * *$ & $-22.41 * *$ \\
\hline 40 & $\mathrm{~L} 43 \times \mathrm{T} 23$ & $-7.58 * *$ & $-23.83 * *$ & $-2.50 *$ & 5.32 & $-8.93 *$ & $-10.03^{*}$ \\
\hline 41 & L43 x T36 & $-35.85 * *$ & $-47.29 * *$ & $-32.00 * *$ & -6.12 & $-19.62 * *$ & $-18.70 * *$ \\
\hline 42 & $\mathrm{~L} 43 \times \mathrm{T} 44$ & $71.72 * *$ & $47.83^{* *}$ & $70.00 * *$ & $52.61 * *$ & $28.43 * *$ & $35.46 * *$ \\
\hline 43 & $\mathrm{~L} 47 \times \mathrm{T} 23$ & $-23.33 * *$ & $-35.16^{* *}$ & $-17.00 * *$ & $-25.01 * *$ & $-29.96 * *$ & $-30.80 * *$ \\
\hline 44 & $\mathrm{~L} 47 \times \mathrm{T} 36$ & $33.33 * *$ & $12.40 * *$ & $45.00 * *$ & $17.47 * *$ & 8.53 & $9.77 *$ \\
\hline 45 & $\mathrm{~L} 47 \times \mathrm{T} 44$ & $-14.50 * *$ & $-24.35^{*}$ & $-13.00 * *$ & $-23.74 * *$ & $-30.87 * *$ & $-27.09 * *$ \\
\hline 46 & $\mathrm{~L} 50 \times \mathrm{T} 23$ & $-38.31 * *$ & $-40.23 * *$ & $-23.50 * *$ & $-41.86 * *$ & $-44.42 * *$ & $-45.09 * *$ \\
\hline 47 & L50 x T36 & $-14.06 * *$ & $-17.05 * *$ & $7.00 * *$ & 3.32 & -2.32 & -1.21 \\
\hline 48 & $\mathrm{~L} 50 \times \mathrm{T} 44$ & $-8.94 * *$ & $-10.83 * *$ & $7.00 * *$ & -5.19 & $-12.09 * *$ & -7.29 \\
\hline 49 & $\mathrm{~L} 53 \times \mathrm{T} 23$ & $-4.93 * *$ & $-17.19 * *$ & $6.00 * *$ & $17.63 * *$ & -0.93 & -2.13 \\
\hline 50 & $\mathrm{~L} 53 \times \mathrm{T} 36$ & $25.00 * *$ & $8.53^{* *}$ & $40.00 * *$ & $45.18 * *$ & $21.12 * *$ & $22.50 * *$ \\
\hline 51 & $\mathrm{~L} 53 \times \mathrm{T} 44$ & $-9.52 * *$ & $-17.39 * *$ & $-5.00 * *$ & $-6.75^{*}$ & $-23.48 * *$ & $-19.30 * *$ \\
\hline 52 & L65 x T23 & $-20.79 * *$ & $-21.88 * *$ & 0.00 & $-16.23 * *$ & $-20.07 * *$ & $-21.03 * *$ \\
\hline 53 & L65 x T36 & $-24.26 * *$ & $-25.58 * *$ & $-4.00 * *$ & $-17.01 * *$ & $-21.68 * *$ & $-20.79 * *$ \\
\hline 54 & L65 x T44 & $-24.01 * *$ & $-26.91 * *$ & $-9.00 * *$ & $-23.73 * *$ & $-29.41 * *$ & $-25.55 * *$ \\
\hline & $\mathrm{SEm} \pm$ & 0.97 & 1.12 & 1.12 & 4.6 & 5.12 & 5.12 \\
\hline & $\mathrm{CD} @ 5 \%$ & 1.95 & 2.25 & 2.25 & 8.4 & 8.80 & 8.80 \\
\hline & CD@1\% & 2.60 & 3.00 & 3.00 & 9.2 & 11.00 & 11.00 \\
\hline
\end{tabular}

Maximum and positive significant heterosis was observed over mid parent $(52.61 \%)$, over better parent $(28.43 \%)$ and over commercial check $(35.46 \%)$ as exhibited by the cross L43 x T44. Maximum heterosis over commercial check for fruit yield per plant was exhibited by the cross L22 × T36 (27.33\%) followed by L22 × T44 (22.94\%), L53 × T36 (22.50\%), L31 × T23 $(21.84 \%), \mathrm{L} 42 \times \mathrm{T} 23(18.55 \%), \mathrm{L} 6 \times \mathrm{T} 44(14.16 \%), \mathrm{L} 14 \times \mathrm{T} 44(11.96 \%), \mathrm{L} 47 \times \mathrm{T} 36(9.77 \%)$ and L17 $\times$ T36 (9.73\%). Among 54 crosses, 16 crosses over mid parent, seven crosses over better parent and 11 crosses over commercial check exhibited positive and significant heterosis. It is pertinent to mention that the crop has potential to produce desired heterotic cross combination which can be further used for yield improvement of this crop. It also indicates that these hybrids may be used for further exploiting heterosis for the improvement of this character. Five crosses, namely, L43 × T44 followed by L22 × T36 (27.33\%), L22 × T44 (22.94\%), L53 × T36 (22.50\%) 
and L31 $\times$ T23 $(21.84 \%)$ were found to be significant for fruit yield per plant (Table 3). On the basis of the findings of this study, and per se performance over both the parents (better parent and economic check) the hybrid L43 x T44 could be exploited commercially for hihger fruit yield. More speiically, in case of fruit yield per hectare among 54 crosses, 23 crosses over mid parent, 12 crosses over better parent and 18 crosses over commercial check showed positive and significant heterosis (Table 4). The finding was expected on in line of significant heterobeltiosis for yield contributing characters as also reported earlier by Chauhan and Singh 2002, Senthil et al. 2005, Ashwani et al. 2013, Kumar et al. 2015, Verma and Sood 2015, More et al. 2015 and Sabesan et al. 2016 in okra.

Table 4. Heterosis (\%) over mid parent, better parent and commercial check for yield/plot and yield per hectare.

\begin{tabular}{|c|c|c|c|c|c|c|c|}
\hline \multirow{2}{*}{$\begin{array}{l}\text { Sl. } \\
\text { No. }\end{array}$} & \multirow{2}{*}{ Crosses } & \multicolumn{3}{|c|}{ Fruit yield per plot } & \multicolumn{3}{|c|}{ Fruit yield per hectare } \\
\hline & & MP & $\mathrm{BP}$ & $\mathrm{CC}$ & MP & $\mathrm{BP}$ & $\mathrm{CC}$ \\
\hline 1 & $\mathrm{~L} 4 \times \mathrm{T} 23$ & $1.32 * *$ & $-16.30 * *$ & $-9.41 * *$ & $1.36^{*}$ & $-16.29 * *$ & $-9.41 * *$ \\
\hline 2 & $\mathrm{~L} 4 \times \mathrm{T} 36$ & $-5.08 * *$ & $-20.00 * *$ & $-17.65 * *$ & $-5.08 * *$ & $-20.02 * *$ & $-17.67 * *$ \\
\hline 3 & $\mathrm{~L} 4 \times \mathrm{T} 44$ & $-21.95 * *$ & $-36.00 * *$ & $-29.41 * *$ & $-21.99 * *$ & $-36.05 * *$ & $-29.47 * *$ \\
\hline 4 & L5 x T23 & $-36.60 * *$ & $-42.93 * *$ & $-38.24 * *$ & $-36.65 * *$ & $-42.97 * *$ & $-38.28 * *$ \\
\hline 5 & L5 x T36 & $-16.21 * *$ & $-22.86 * *$ & $-20.59 * *$ & $-16.24 * *$ & $-22.88 * *$ & $-20.61 * *$ \\
\hline 6 & L5 x T44 & $4.56^{* *}$ & $-6.67 * *$ & $2.94 * *$ & $4.56^{* *}$ & $-6.66 * *$ & $2.94 * *$ \\
\hline 7 & L6 x T23 & $-1.11 * *$ & $-3.53 * *$ & $4.41 * *$ & -1.11 & $-3.52 * *$ & $4.41 * *$ \\
\hline 8 & L6 x T36 & $-45.71 * *$ & $-45.71 * *$ & $-44.12 * *$ & $-45.76 * *$ & $-45.76 * *$ & $-44.16 * *$ \\
\hline 9 & L6 x T44 & $4.97 * *$ & $1.33 * *$ & $11.76^{* *}$ & $4.84 * *$ & $1.36^{* *}$ & $11.75 * *$ \\
\hline 10 & $\mathrm{~L} 14 \times \mathrm{T} 23$ & $2.42 * *$ & $-16.58 * *$ & $-9.71 * *$ & $2.43 * *$ & $-16.59 * *$ & $-9.73 * *$ \\
\hline 11 & L14 x T36 & $-27.77 * *$ & $-40.00 * *$ & $-38.24 * *$ & $-27.80 * *$ & $-40.05 * *$ & $-38.28 * *$ \\
\hline 12 & L14 x T44 & $4.97 * *$ & $1.33^{* *}$ & $11.76 * *$ & $4.84 * *$ & $1.36^{* *}$ & $11.75 * *$ \\
\hline 13 & $\mathrm{~L} 16 \times \mathrm{T} 23$ & 0.31 & $-13.04 * *$ & $-5.88 * *$ & 0.34 & $-13.03 * *$ & $-5.88 * *$ \\
\hline 14 & L16 x T36 & $-6.45 * *$ & $-17.14 * *$ & $-14.71 * *$ & $-6.43 * *$ & $-17.13 * *$ & $-14.69 * *$ \\
\hline 15 & $\mathrm{~L} 16 \times \mathrm{T} 44$ & $23.26 * *$ & $6.00 * *$ & $16.91 * *$ & $23.28 * *$ & $6.01 * *$ & $16.92 * *$ \\
\hline 16 & L17 x T23 & $3.11 * *$ & $-7.61 * *$ & 0.00 & $3.11 * *$ & $-7.60 * *$ & 0.00 \\
\hline 17 & L17 x T36 & $21.59 * *$ & $11.43 * *$ & $14.71 * *$ & $21.57 * *$ & $11.42 * *$ & $14.69 * *$ \\
\hline 18 & $\mathrm{~L} 17 \times \mathrm{T} 44$ & $5.03 * *$ & $-6.67 * *$ & $2.94 * *$ & $5.02 * *$ & $-6.66 * *$ & $2.94 * *$ \\
\hline 19 & $\mathrm{~L} 22 \times \mathrm{T} 23$ & $-20.41 * *$ & $-31.93 * *$ & $-26.32 * *$ & $-20.40 * *$ & $-31.93 * *$ & $-26.33 * *$ \\
\hline 20 & L22 x T36 & $40.90 * *$ & $22.85 * *$ & $26.47 * *$ & $40.63 * *$ & $22.83 * *$ & $26.44 * *$ \\
\hline 21 & $\mathrm{~L} 22 \times \mathrm{T} 44$ & $30.50 * *$ & $10.66 * *$ & $22.05 * *$ & $30.47 * *$ & $10.73 * *$ & $22.08 * *$ \\
\hline 22 & L24 x T23 & $-25.84 * *$ & $-33.70 * *$ & $-28.24 * *$ & $-25.84 * *$ & $-33.69 * *$ & $-28.24 * *$ \\
\hline 23 & L24 x T36 & $-7.19 * *$ & $-15.14 * *$ & $-12.65 * *$ & $-7.22 * *$ & $-15.16^{* *}$ & $-12.67 * *$ \\
\hline 24 & $\mathrm{~L} 24 \times \mathrm{T} 44$ & $-12.03 * *$ & $-22.00 * *$ & $-13.97 * *$ & $-7.98 * *$ & $-18.40 * *$ & $-10.01 * *$ \\
\hline 25 & $\mathrm{~L} 31 \times \mathrm{T} 23$ & $12.99 * *$ & $8.69 * *$ & $17.64 * *$ & $13.05 * *$ & $8.73 * *$ & $17.63 * *$ \\
\hline 26 & L31 x T36 & $7.54 * *$ & $6.00 * *$ & $9.12 * *$ & $7.51 * *$ & $5.98 * *$ & $9.09 * *$ \\
\hline 27 & $\mathrm{~L} 31 \times \mathrm{T} 44$ & $-18.46^{* *}$ & $-22.27 * *$ & $-14.26^{* *}$ & $-18.45 * *$ & $-22.25 * *$ & $-14.26 * *$ \\
\hline 28 & L37 x T23 & $17.10 * *$ & $-14.40 * *$ & $-7.35^{* *}$ & $17.15^{* *}$ & $-14.39 * *$ & $-7.35 * *$ \\
\hline 29 & L37 x T36 & $3.85^{* *}$ & $-22.86 * *$ & $-20.59 * *$ & $3.84 * *$ & $-22.88 * *$ & $-20.61 * *$ \\
\hline 30 & L37 x T44 & $-19.27 * *$ & $-41.33 * *$ & $-35.29 * *$ & $-19.31 * *$ & $-41.38 * *$ & $-35.35 * *$ \\
\hline 31 & $\mathrm{~L} 38 \times \mathrm{T} 23$ & $2.08 * *$ & $-3.53 * *$ & $4.41 * *$ & $2.06^{* *}$ & $-3.56 * *$ & $4.37 * *$ \\
\hline 32 & L38 x T36 & $-32.10 * *$ & $-34.29 * *$ & $-32.35 * *$ & $-32.15 * *$ & $-34.34 * *$ & $-32.41 * *$ \\
\hline
\end{tabular}


Contd.

\begin{tabular}{|c|c|c|c|c|c|c|c|}
\hline \multirow{2}{*}{$\begin{array}{l}\text { Sl. } \\
\text { No. }\end{array}$} & \multirow[t]{2}{*}{ Crosses } & \multicolumn{3}{|c|}{ Fruit yield per plot } & \multicolumn{3}{|c|}{ Fruit yield per hectare } \\
\hline & & MP & $\mathrm{BP}$ & $\mathrm{CC}$ & MP & $\mathrm{BP}$ & $\mathrm{CC}$ \\
\hline 33 & $\mathrm{~L} 38 \times \mathrm{T} 44$ & $-25.98 * *$ & $-30.67 * *$ & $-23.53 * *$ & $-25.99 * *$ & $-30.68 * *$ & $-23.55^{* *}$ \\
\hline 34 & L39 x T23 & $7.67 * *$ & $-0.82 * *$ & $7.35^{* *}$ & $7.67 * *$ & -0.81 & $7.35^{* *}$ \\
\hline 35 & L39 x T36 & $-24.24 * *$ & $-28.57 * *$ & $-26.47 * *$ & $-24.30 * *$ & $-28.63 * *$ & $-26.53 * *$ \\
\hline 36 & L39 x T44 & $0.73 * *$ & $-8.00 * *$ & $1.47 * *$ & 0.73 & $-7.99 * *$ & $1.47^{*}$ \\
\hline 37 & $\mathrm{~L} 42 \times \mathrm{T} 23$ & $27.39 * *$ & $8.70 * *$ & $17.65^{* *}$ & $27.40 * *$ & $8.70 * *$ & $17.63 * *$ \\
\hline 38 & $\mathrm{~L} 42 \times \mathrm{T} 36$ & $-37.70 * *$ & $-45.71 * *$ & $-44.12 * *$ & $-37.75 * *$ & $-45.76 * *$ & $-44.16 * *$ \\
\hline 39 & $\mathrm{~L} 42 \times \mathrm{T} 44$ & $-8.66 * *$ & $-22.67 * *$ & $-14.71 * *$ & $-8.63 * *$ & $-22.65 * *$ & $-14.69 *$ \\
\hline 40 & $\mathrm{~L} 43 \times \mathrm{T} 23$ & $11.77 * *$ & $10.33 * *$ & $-2.94 * *$ & $11.80 * *$ & $-10.31 * *$ & $-2.94 * *$ \\
\hline 41 & $\mathrm{~L} 43 \times \mathrm{T} 36$ & $7.25 * *$ & $-12.29 * *$ & $-9.71 * *$ & $7.24 * *$ & $-12.31 * *$ & $-9.73 * *$ \\
\hline 42 & $\mathrm{~L} 43 \times \mathrm{T} 44$ & $51.00 * *$ & $20.00 * *$ & $32.35 * *$ & $50.76 * *$ & $20.02 * *$ & $32.32 * *$ \\
\hline 43 & $\mathrm{~L} 47 \times \mathrm{T} 23$ & $-30.94 * *$ & $-34.78 * *$ & $-29.41 * *$ & $-30.99 * *$ & $-34.83 * *$ & $-29.47 * *$ \\
\hline 44 & L47 x T36 & $9.76^{* *}$ & $6.00 * *$ & $9.11 * *$ & $9.65^{* *}$ & $6.01 * *$ & $9.13 * *$ \\
\hline 45 & $\mathrm{~L} 47 \times \mathrm{T} 44$ & $-25.93 * *$ & $-30.67 * *$ & $-23.53 * *$ & $-25.95 * *$ & $-30.68 * *$ & $-23.55 * *$ \\
\hline 46 & $\mathrm{~L} 50 \times \mathrm{T} 23$ & $-39.27 * *$ & $-41.58 * *$ & $-36.76 * *$ & $-39.19 * *$ & $-41.61 * *$ & $-36.81 * *$ \\
\hline 47 & L50 x T36 & $-17.25 * *$ & $-18.43 * *$ & $-16.03 * *$ & $-17.10 * *$ & $-18.44 * *$ & $-16.04 * *$ \\
\hline 48 & $\mathrm{~L} 50 \times \mathrm{T} 44$ & $-33.57 * *$ & $-36.67 * *$ & $-30.15^{* *}$ & $-33.47 * *$ & $-36.69 * *$ & $-30.18 * *$ \\
\hline 49 & $\mathrm{~L} 53 \times \mathrm{T} 23$ & $-31.01 * *$ & $-38.18 * *$ & $-33.09 * *$ & $-31.04 * *$ & $-38.20 * *$ & $-33.12 * *$ \\
\hline 50 & L53 x T36 & $26.56 * *$ & $15.71 * *$ & $19.11 * *$ & $26.56 * *$ & $15.71 * *$ & $19.11 * *$ \\
\hline 51 & L53 x T44 & $-12.98 * *$ & $-22.67 * *$ & $-14.71 * *$ & $-12.97 *$ & $-22.65 * *$ & $-14.69 * *$ \\
\hline 52 & L65 x T23 & $-22.32 * *$ & $-25.27 * *$ & $-19.12 * *$ & $-22.19 * *$ & $-25.28 * *$ & $-19.14 * *$ \\
\hline 53 & L65 x T36 & $-18.84 * *$ & $-20.00 * *$ & $-17.65^{* *}$ & $-18.71 * *$ & $-20.02 * *$ & $-17.67 * *$ \\
\hline \multirow[t]{4}{*}{54} & L65 x T44 & $-32.31 * *$ & $-35.47 * *$ & $-28.82 * *$ & $-32.19 * *$ & $-35.47 * *$ & $-28.83 * *$ \\
\hline & SEm \pm & 0.15 & 0.18 & 0.18 & 0.62 & 0.71 & 0.71 \\
\hline & $\mathrm{CD} @ 5 \%$ & 0.31 & 0.36 & 0.36 & 1.24 & 1.44 & 1.44 \\
\hline & CD@1\% & 0.42 & 0.48 & 0.48 & 1.66 & 1.92 & 1.92 \\
\hline
\end{tabular}

Overall the study revealed that the magnitude of percentage over commercial check were found more or less similar among all the characters of okra. Out of 54 crosses five crosses, namely $\mathrm{L} 43 \times \mathrm{T} 44, \mathrm{~L} 22 \times \mathrm{T} 36, \mathrm{~L} 22 \times \mathrm{T} 44, \mathrm{~L} 53 \times \mathrm{T} 36$ and L31 $\times \mathrm{T} 23$ were found potential for the production of fruit yield per plant and other desired characters. The high heterosis and per se performance was found in hybrid L43 $\times$ T44 over both better parents and standard check for fruit yield per plant. This indicates that the cross can be exploited commercially for yield enhancement in okra.

\section{References}

Anonymous 2016a. Indian Horticultural Database. http://www.nhb.gov.in

Ashwani K, Baranwal DK, Aparna J and Srivastava K 2013. Combining ability and heterosis for yield and contributing characters in okra [Abelmoschus esculentus (L.) Moench]. Madras Agric. J. 100(1-3): 30-35.

Aykroyd, W. R., 1963, ICMR Special Report New Delhi. Series No. 42.

Chauhan S and Singh Y 2002. Heterosis studies in okra [Abelmoschus esculentus (L.) Moench]. Veg. Sci. 29(2): 116-118. 
Duggi S, Magadum S, Kishor DS, Srinivasraghavan A, Sunny KO and Arya K 2013. Screening of okra [Abelmoschus esculentus (L.) Moench] genotypes for shoot and fruit borer resistance. Bionfolet. 10(2b): 653-657.

Jindal SK, Aror D and Ghai TR 2009. Heterobelotiosis and combining ability for earliness in okra [Abelmoschus esculentus (L.) Moench.). Crop Improv. 36(1): 1-5.

Khanpara MD, Jivani LL, Vachhani JH, Shekhat HG and Mehta DR 2009. Line $\times$ tester analysis for combining ability in okra [Abelmoschus esculentus (L.) Moench]. Int. J. Agricul. Sci. 5(2): 554-557.

Kempthorne, O., 1957, The theory of diallel crosses. Genetics, 41: 451-459.

Kishor DS, Arya K, Duggi S, Magadum S, Raghavendra NR, Challa V and Reddy PS 2013. Studies on heterosis for yield and yield contributing traits in okra [Abelmoschus esculentus (L.) Moench]. Molecular Plant Breed. 4(35): 277-284.

Kumar N, Saravaiya SN, Patel AI and Nazaneen NS 2015. Heterosis studies in okra (Abelomoschus esculantus (L.) Moench). Trends in Biosci. 8(1): 236-241.

More SJ, Chaudhari KN, Bhanderi DR, Saravaiya SN and Chawla SL 2015. Heterosis study in okra (Abelmoschus esculentus (L.) Moench). Trends in Biosci. 8(12): 3552-3255.

Neetu Singh AK, Kumar R and Pal M 2015. Heterosis and inbreeding depression in okra (Abelomoschus esculentus (L.) Moench). Int. J. Scientific and Innovative Res. 32(2): 15-24.

Sabesan T, Sarvanan K and Satheeshkumar P 2016. Studies on heterosis, inbreeding depression and residual heterosis for fruit yield and its components in okra (Abelmoschus esculentus (L.) Moench). Plant Archive 16(2): 669-674.

Senthil NK, Kumar ST, Saravanan, K and Ganesan J 2005. Heterosis and inbreeding depression for yield and yield components in bhendi [Ablemoschus esculentus (L.) Moench]. Agric. Sci. Digest. 25 (2):142-144.

Singh AK and Singh MC 2012. Studies of heterosis and identification of superior crosses in okra (Abelmoschus esculentus (L.) Moench). Agric. Sci. Digest. 32(1): 55-57.

Shoba K and Mariappan S 2005, Studies on heterosis for growth, flowering, fruit characters in okra [Abelmoschus esculentus (L.) Moench]. South Indian Hort. 53(1-6): 303-305.

Thippeswamy S 2001. Line $\mathrm{x}$ tester analysis for heterosis and combining ability using male sterile lines in okra. M. Sc. (Agri.) Thesis, Univ. Agril. Sci., Bangalore.

Verma A and Sood S 2015. Genetic expression of heterosis for yield and yieldcomponents in intraspecific hybrids of okra (Abelmoschus esculentus (L.) Moench). SABRO J. Breed. Genet. 47(3):221-230.

Vijayaraghavan C and Warier UA 1946. Evaluation of high yielding bhendi (Hibiscu sesculentus). Proc. 33rd Indian Sci. Cong. 33: 165. 